\title{
Bioinformatics analysis identifies potential chemoresistance-associated genes across multiple types of cancer
}

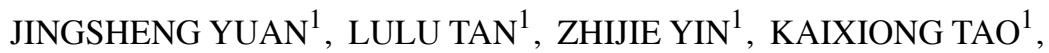 \\ GUOBING WANG $^{1}$, WENJIA SHI ${ }^{2 *}$ and JINBO GAO ${ }^{{ }^{*}}$ \\ Departments of ${ }^{1}$ Gastrointestinal Surgery and ${ }^{2}$ Pediatric Surgery, Union Hospital, Tongji Medical College, \\ Huazhong University of Science and Technology, Wuhan, Hubei 430022, P.R. China
}

Received July 10, 2018; Accepted May 13, 2019

DOI: $10.3892 / \mathrm{ol} .2019 .10533$

\begin{abstract}
Despite the fact that studies have revealed mechanisms underlying tumor chemoresistance, the functions of numerous potential chemoresistance-associated genes have yet to be elucidated. A bioinformatics analysis was conducted to screen differentially expressed genes (DEGs) across four types of chemoresistant tumors and functional enrichment analysis was used to examine the biological significance of these genes. Furthermore, a gene network was constructed using weighted gene co-expression network analysis to identify hub genes. A total of $6,015,2,074,2,141$ and 954 differentially expressed genes were identified in estrogen receptor-negative breast cancer, ovarian cancer, rectal cancer and gastric cancer, respectively; however, only five of these DEGs were dysregulated in all four types of cancer. Functional enrichment analysis of the DEGs suggested that genomic stability and immune response are crucial determinants of tumor chemoresistance. In addition, 14, 8, 6 and 1 co-expressed gene modules were identified in estrogen receptor-negative breast cancer, ovarian cancer, rectal cancer and gastric cancer, respectively, and protein-protein interaction networks were created. The analysis identified only calcium-calmodulin-dependent protein kinase kinase 2, erythropoietin receptor, mitochondrial poly(A) RNA polymerase, $\alpha$-parvin, and zinc finger and BTB domain-containing protein 44 to be dysregulated in all four cancer types, indicating varying
\end{abstract}

Correspondence to: Professor Jinbo Gao, Department of Gastrointestinal Surgery, Union Hospital, Tongji Medical College, Huazhong University of Science and Technology, 1,277 Jiefang Avenue, Wuhan, Hubei 430022, P.R. China

E-mail: jgao@hust.edu.cn

Dr Wenjia Shi, Department of Pediatric Surgery, Union Hospital, Tongji Medical College, Huazhong University of Science and Technology, 1,277 Jiefang Avenue, Wuhan, Hubei 430022, P.R. China E-mail:wshi@hust.edu.cn

*Contributed equally

Key words: bioinformatics, chemoresistance, cancer, weighted gene co-expression network analysis, network-based screening mechanisms of chemoresistance in different tumor types. Furthermore, our analysis suggests that type I collagen $\alpha 1$, fibroblast growth factor 14 and major histocompatibility complex, class II, DR $\beta 1$ potentially serve key roles in the development of chemoresistance. In conclusion, the present study proposes a simple and effective strategy for identifying genes involved in chemoresistance and predicting their potential functional roles, which may guide subsequent experimental studies.

\section{Introduction}

The main obstacle to successful chemotherapy is multidrug resistance, which involves the development of resistance to multiple chemotherapeutic agents with different structures and mechanisms of action following exposure to a single chemotherapeutic agent or even upon initial exposure (1). Tumor chemotherapy resistance is a complex multifactor-mediated process, and the numerous mechanisms involved include abnormal expression of ATP-dependent transporters on the cell membrane, enhancement of cell detoxification and dysregulation of apoptosis-associated mechanisms $(2,3)$. A thorough understanding of the mechanisms of tumor resistance may provide a tool for overcoming the aforementioned, and based on a growing body of research into the genetic diversity among chemotherapy-resistant cell lines and clinical resistance, tumor chemosensitivity appears to be controlled by complex molecular networks.

Network approaches have been employed to study the development of various diseases, such as cancer and metabolic disease, bridging the gap from individual genes to the occurrence and development of a disease by examining the associations between differentially expressed genes (DEGs) $(4,5)$. In the present study, bioinformatics analysis was used to reveal common properties of DEGs between chemoresistant and chemosensitive tumors across multiple cancers. A total of five co-expressed genes were identified and their biological functions examined. In addition, weighted gene co-expression network analysis (WGCNA) was applied and 14 modules were identified in estrogen receptor-negative breast cancer (EBC), 8 in ovarian cancer (OVC), 6 in rectal cancer (RC), and 1 in gastric cancer (GC), and the differences in these modules among these tumors were examined. Therefore, the present study proposes a systematic context for identifying candidate genes that may be useful in the study of chemoresistance. 
Furthermore, the construction of a co-expression network of weighted genes is an effective approach for associating differences in gene expression with insights into key functional chemosensitivity mechanisms.

\section{Materials and methods}

Microarray data and differential expression analysis. To investigate the genes involved in chemoresistance, Gene Expression Omnibus (GEO) database was searched comprehensively, and microarray data from human EBC, OVC, RC and GC were collected from four published studies (6-9). All raw data from these studies consist of RNA sequencing (RNA-Seq) datasets for the study of tumor chemotherapeutic resistance and are available in Gene Expression Omnibus (GEO), including 120 samples in GSE16446 (6), 58 samples in GSE30161 (7), 80 samples in GSE45404 (8) and 44 samples in GSE14209 (9) (ncbi.nlm. nih.gov/geo/). EBC, OVC and RC samples were analyzed using Affymetrix HG-U133_Plus_2 microarrays (ncbi.nlm. nih.gov/geo/query/acc.cgi?acc=GPL570); GC samples were analyzed using Affymetrix HG-U133A_2 microarrays (ncbi. nlm.nih.gov/geo/query/acc.cgi?acc=GPL571). Samples where the chemotherapy effects were not clearly stated were removed.

The GSE16446, GSE30161, GSE45404 and GSE14209 datasets were screened using the GEO2R tool (ncbi.nlm.nih. gov/geo/geo2r/) to identify DEGs between chemoresistant and chemosensitive tumors (6-9). $\mathrm{P}<0.05$ was used to define a gene as a DEG.

Functional enrichment analysis. Database for Annotation, Visualization and Integrated Discovery (DAVID) v6.8 (david-d. ncifcrf.gov/) was used to analyze functional enrichment among DEGs $(10,11)$. Four representative terms from the top 10 most significant Gene Ontology (GO) biological process (BP) terms were analyzed for each cancer. In addition, only those Kyoto Encyclopedia of Genes and Genomes (KEGG) pathways with $\mathrm{P} \leq 0.05$ and $\geq 10$ enriched genes were considered significant.

WGCNA. For each dataset, chemoresistant samples and DEGs were firstly checked with many missing values and the samples were subsequently clustered to identify obvious outliers. Following the removal of such samples, Pearson correlation coefficients were calculated for all pairwise comparisons of DEGs. A similarity (correlation) matrix was obtained using a power function (connection strength $=$ |correlation $\left.\right|^{\beta}$ ), and a WGCNA was constructed with power values of 12 in EBC, 8 in OVC, 8 in RC and 5 in GC. Modules were obtained from four types of tumors using a dynamic tree-cutting algorithm (12), and genes that were not assigned to modules were assigned the color gray. Due to the large amount of genetic analysis, additional steps were performed for each module, and WGCNA was employed to construct protein-protein interaction (PPI) networks based on the signed Pearson (12). Detailed R software code (https://labs.genetics.ucla.edu/ horvath/htdocs/CoexpressionNetwork/Rpackages/WGCNA/) and tutorials were used for generating co-expression networks for weighted genes (12).

Statistical analysis and visualization. Statistical analysis was performed using R-3.3.1 (https://www.r-project.org). The majority of visualizations were generated using $\mathrm{R}$, except for KEGG network visualization, for which the (version 3.3.0; cytoscape.org/) and ClueGO tools (version 2.5.0) were used $(13,14)$. For visualization of KEGG networks, the top five (top four for GC) were selected for each cancer to analyze pivotal links between KEGG pathways.

\section{Results}

DEGs. DEGs between chemoresistant and -sensitive samples were analyzed based on the criterion of $\mathrm{P}<0.05$ and a total of 6,015, 2,074, 2,141 and 954 DEGs were identified in EBC, OVC, RC and GC, respectively. Hierarchical cluster analysis was also performed to obtain an overview of the profile for DEGs in each cancer (Fig. 1). All heat maps revealed separation if DEGs between chemoresistant and chemosensitive samples.

Among all DEGs, 1,335 genes were differentially expressed between any two of the cancer types. Only 121 DEGs were altered in more than two types of cancer (Fig. 2A). Among these 121 DEGs, the following five were dysregulated in all four cancer types examined (Fig. 2A): Calcium-calmodulin-dependent protein kinase kinase 2 (CaMKK2), erythropoietin receptor (EPOR), mitochondrial poly(A) RNA polymerase (MTPAP), $\alpha$-parvin (PARVA) and zinc finger and BTB domain-containing protein 44 (ZBTB44).

Functional enrichment analysis. To reveal the biological significance of DEGs in regulating cancer chemoresistance at the unitary level, DAVID v6.7 was used to perform biofunctional enrichment analysis for each tumor type, including GO BP terms, GO cellular component terms, GO molecular functions terms and KEGG pathways. Fig. 2B displays four representative BP terms for each tumor type. Fig. 3 lists the top 10 KEGG pathways with $\mathrm{P} \leq 0.05$ and enriched gene counts $\geq 10$ for each tumor type.

The DEGs were divided into two sections according to major biological processes. On the one hand, as observed in Fig. 2B, the DEGs in the four tumors were significantly enriched in processes associated with DNA replication, transcription and regulation of these pathways. On the other hand, a number of chemoresistance-associated genes were enriched in pathways involved in immune activity and stimulation, including pathways associated with antigen processing and presentation via MHC class II and negative regulation of megakaryocyte differentiation. Based on the cellular component and molecular function terms that DEGs' were enriched in (data not shown), the main functional sites and specific effects of the DEGs appear to be involved in the regulation of tumor chemotherapy resistance.

DAVID-based enrichment analysis identified KEGG pathways for the DEGs among the four types of tumors (Fig. 3), including some carcinogenic signaling pathways, including the Notch, RAP1 GTPase-activating protein 1, mitogen-activated protein kinase (MAPK) and PI3K-AKT pathways. Despite that associations between these pathways and chemoresistance have already been established (15-17), the approach of the present analysis systematically identified the biological functions of DEGs involved. In addition, within defined KEGG pathways, immune response-associated pathways were indicated to be 

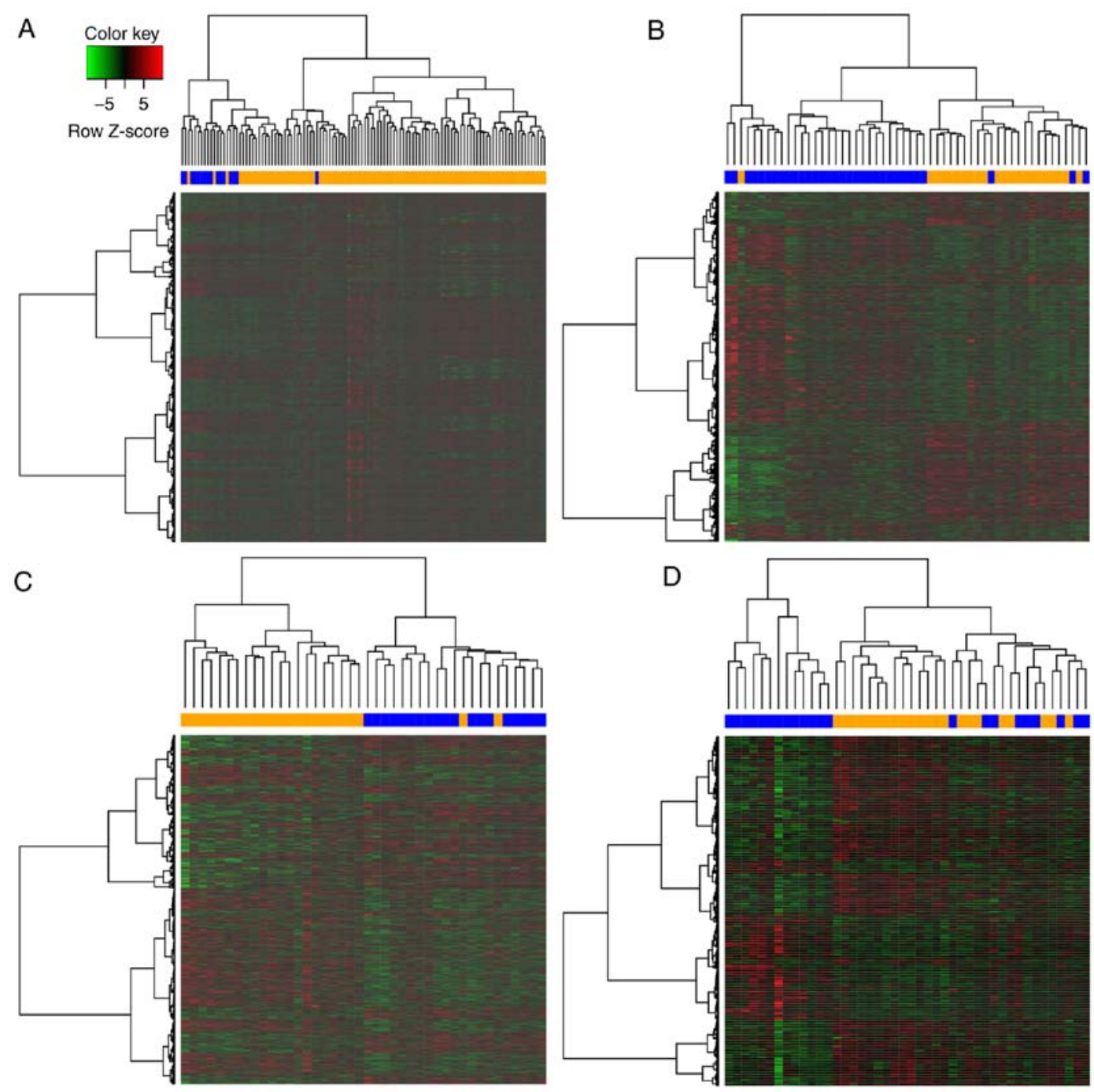

Figure 1. Hierarchical cluster analysis based on DEGs for each cancer type. (A) The heatmap of 6,015 DEGs in estrogen receptor-negative breast cancer. (B) The heatmap of 2,074 DEGs in ovarian cancer. (C) The heatmap of 2,141 DEGs with rectal cancer. (D) The heatmap of 954 DEGs in gastric cancer. The color bar of the heatmap represents the sample groups: Blue indicates a chemosensitive sample, and orange represents a chemoresistant sample. DEGs, differentially expressed genes.
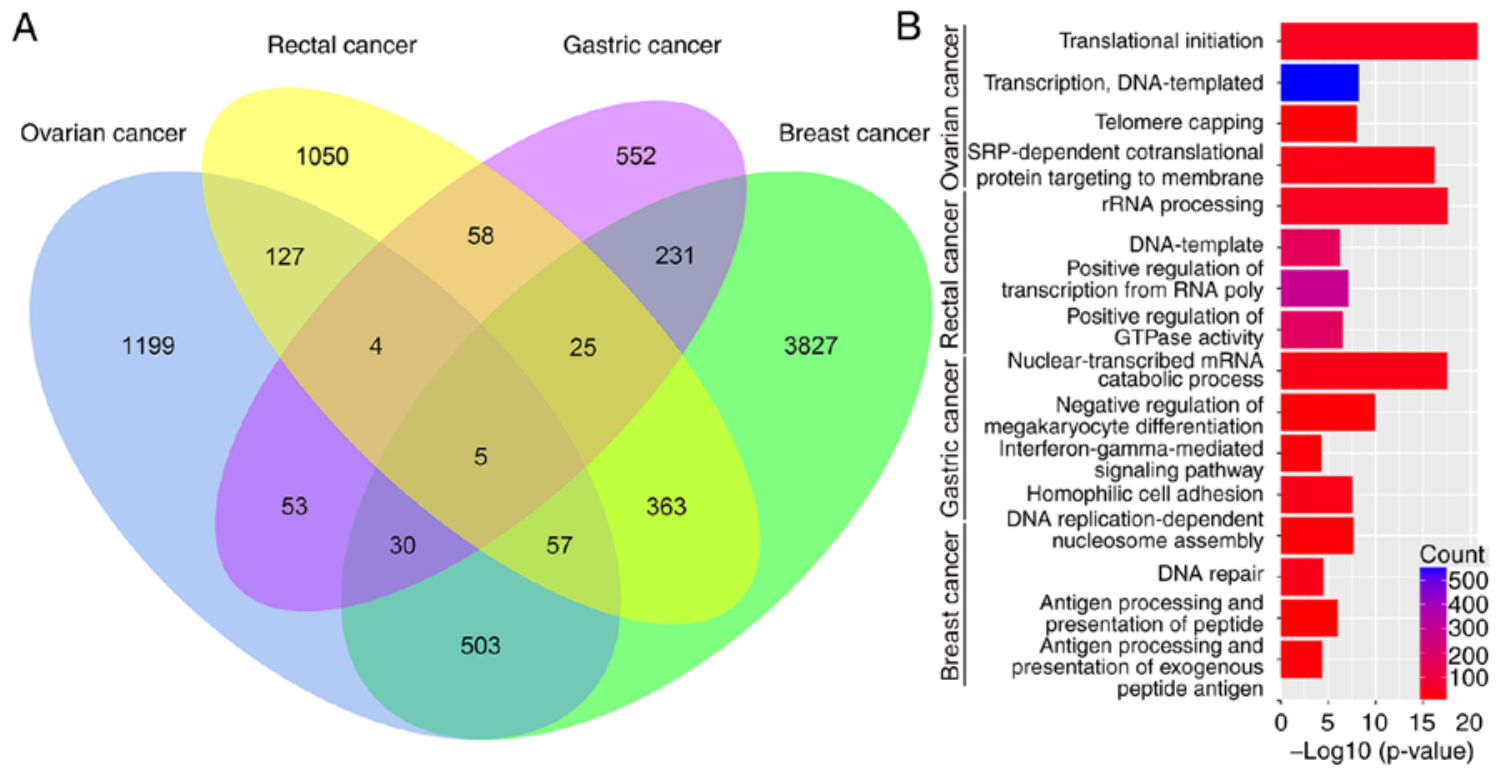

Figure 2. (A) Venn plot diagram of differentially expressed genes in four cancer types. (B) The four representative terms of biological processes for each tumor type. rRNA, ribosomal RNA; SRP, signal-recognition particle. 
A

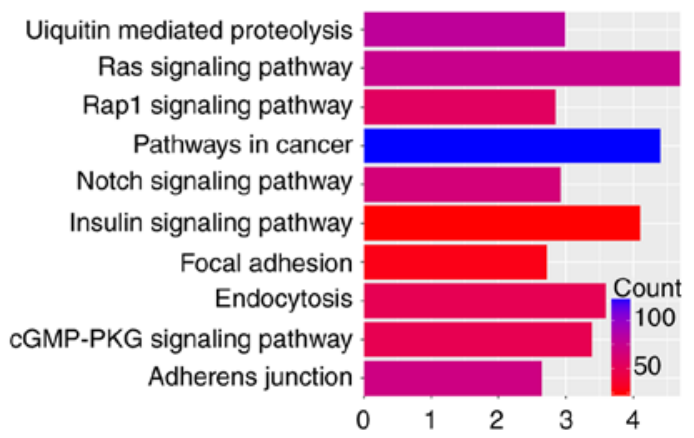

C

Transcriptional misregulation Intestinal immune network for IgA production Jak-STAT signaling pathway Antigen processing and presentation Cell adhesion molecules (CAMs) Phagosome p53 signaling pathway Cell cycle

FoxO signaling pathway ErbB signaling pathway

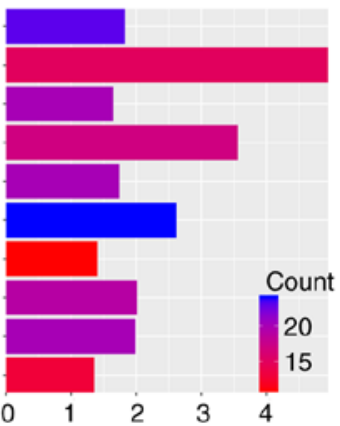

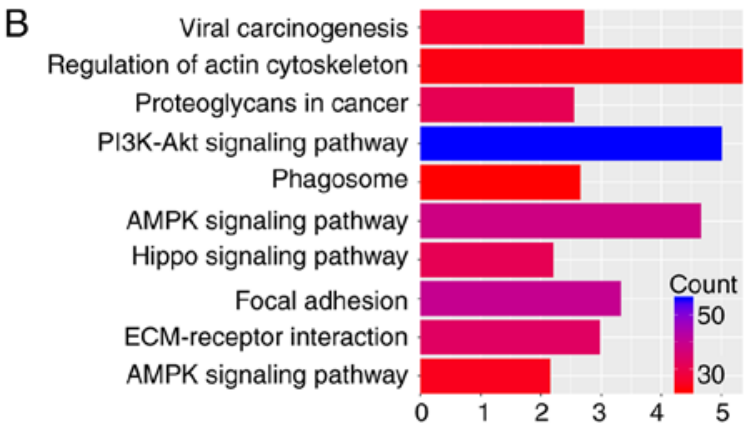

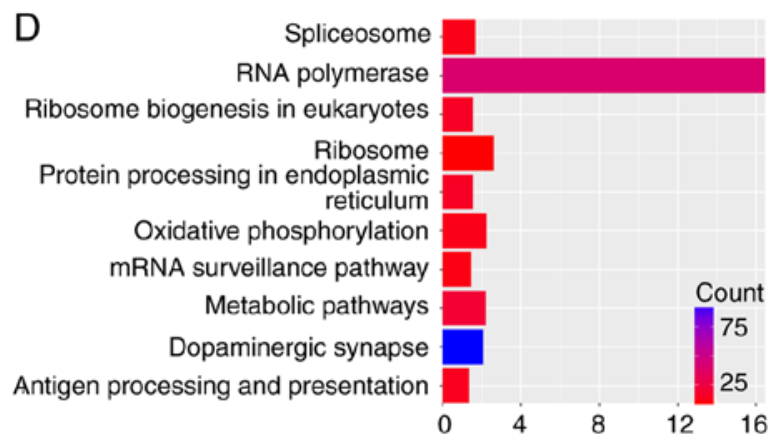

Figure 3. The top 10 Kyoto Encyclopedia of Genes and Genomes pathways in (A) estrogen receptor-negative breast cancer, (B) ovarian cancer, (C) rectal cancer (D) and gastric cancer.

associated with chemoresistance in tumors via processes, including antigen processing and presentation. In particular, different mechanisms of chemoresistance were identified in the individual tumor types. For instance, cell adhesion, including extracellular matrix (ECM)-receptor interactions and focal adhesions, was indicated to be associated with the regulation of drug resistance in OVC. To further visualize these associations between the DEGs in KEGG pathways, a KEGG signaling pathway map was generated, which revealed that a number of genes constitute pivotal links between KEGG pathways (Fig. 4). In addition, it was indicated that nodes involved in the formation of tumor chemoresistance are simultaneously involved in multiple KEGG pathways (Table I).

Gene co-expression networks. Identifying genes with highly correlated expression levels may help elucidate shared biological processes or common regulatory mechanisms that could be targeted $(18,19)$. Therefore, DEGs between chemoresistant and chemosensitive tumors were investigated by applying WGCNA to microarray datasets for four types of tumors. To ensure high-quality data, strict quality control procedures were implemented to eliminate non-specific and mis-targeted probes on the microarray and to remove outlier samples from the dataset prior to generating expression values (data not shown). To facilitate the identification of gene modules (groups of highly co-expressed genes), a WGCN based on pairwise Pearson correlations was constructed for DEGs for these four types of cancer. The WGCNA was intended to identify modules with highly topologically overlapping co-expressed genes, and a pairwise approach was applied to describe the similarity of two co-expressed genes with all other genes in the network. The highest number of modules for gene co-expression was for EBC samples, with 14 genes; in contrast, the lowest number of modules was obtained for GC samples, with only one. Eight and six gene co-expression modules were generated for OVC and RC samples, respectively (Fig. 5). Each model across the four types of tumors included $\geq 30$ genes.

Highly relevant hub genes are thought to serve an important role in the biological behavior of tissues, and central hub genes in a network are more likely to be key drivers of aberrant behavior compared with peripheral genes $(12,20)$. Therefore, the hub genes were identified for each module of the four tumor types and the top 30 nodes for that module were subsequently selected with the highest degree as the central gene. To visualize these results, a list of the network diagrams of these hub genes was compiled for each of the modules (data not shown). The centrality of the 'hub' genes obtained indicates that they may serve roles that are more crucial in the generation of chemoresistance compared with other genes in that module. The gene with the highest degree from each module is listed in Table II. Among them, the hub gene type I collagen $\alpha 1$ (COL1A1) is involved in the PI3K-AKT signaling pathway, focal adhesions and ECM-receptor interactions. The hub gene fibroblast growth factor 14 (FGF14) is associated with both the PI3K-AKT signaling pathway and the MAPK signaling pathway. The hub gene major histocompatibility complex, class II, DR $\beta 1$ (HLA-DRB1) is associated with the phagosome, antigen processing and presentation and the intestinal immune network for IgA production. In addition, MTPAP was differentially expressed among the four tumors and was also the hub gene in one of the models.

\section{Discussion}

Although an increasing number of studies have revealed the important roles served by certain genes and signaling pathways in the development of tumor chemoresistance (21-24), the roles of a number of potential chemoresistance-associated genes 


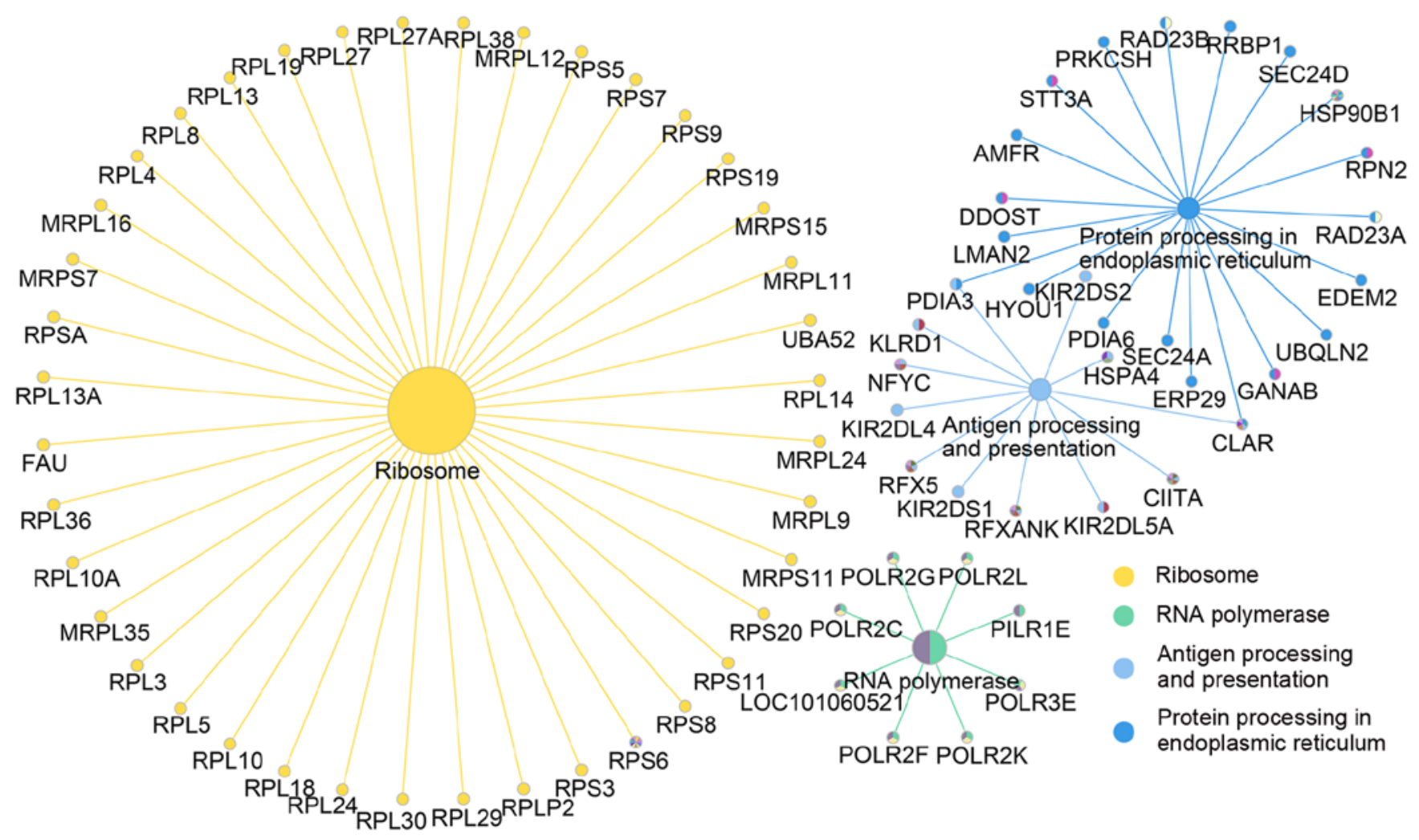

Figure 4. Map of the DEGs associated with the top four Kyoto Encyclopedia of Genes and Genomes signaling pathways in gastric cancer. Maps for estrogen receptor-negative breast cancer, ovarian cancer and rectal cancer are not shown. Different colors indicate different gene clusters.

have not yet been uncovered. The purpose of the current study was to mine the RNA-Seq data from patients with chemoresistant tumors in GEO using WGCNA to identify potential genes associated with chemoresistance. DEGs were screened for four types of chemoresistant tumors using the GEO2R tool and functional enrichment analysis was used to examine the biological importance of the DEGs identified. Furthermore, a gene network was constructed using WGCNA to identify hub genes.

The majority of the DEGs identified in this study, to the best of our knowledge, have not been previously reported to be associated with chemotherapy-resistant cancers. During differential expression analysis, it was indicated that only five genes were differentially expressed in all four types of tumors, indicating varying mechanisms of chemoresistance among tumor types. When consulting previous studies, it was indicated that all five of these oncogenes have been reported to be involved in tumorigenesis. In prostate cancer, the CaMKK2-AMP-activated protein kinase axis controls glucose metabolism, tumor cell growth and migration (25). A number of studies have also reported that CaMKK2 is located at the top of the key molecular node that shapes the cancer ecosystem, highlighting that it is a potential candidate for therapeutic cancer intervention $(25,26)$. Studies of EPOR signaling cascades in tumor cells have revealed that numerous patients with cancer are anemic, with persistent and worsening effects (27). MTPAP governs 3' nucleotide addition to microRNAs (miRNAs) in a miRNA-specific manner and can influence miRNA stability and efficiency for target repression (28). PARVA localizes to focal adhesions and serves critical roles in a number of cellular functions, including regulation of cytoskeletal activity in connection with the ECM, with involvement by paxillin and integrin-linked kinase (29). The transcriptional regulator ZBTB44 interacts with Smad pathway proteins, including Smad-specific E3 ubiquitin ligase 2, mediating resistance to MAPK pathway inhibitors (30). However, these five DEGs have only been studied in individual cancer types; therefore, no experimental evidence or clinical data, to the best of our knowledge, exist to support their associations with cancer chemoresistance. The expression levels of individual genes differed across the four types of tumor, again confirming that the same gene may induce chemoresistance through differential mechanisms in different tumors. A limitation of the present study however is that these five DEGs were not further validated in cells or tissues.

According to functional enrichment analysis, multiple processes associated with gene expression and regulation and classical tumor signaling pathways were enriched, including many carcinogenic signals (31). These findings indicate that aberrant growth signals regulate the cell cycle and cell growth $(32,33)$, and also promote chemotherapy resistance. Research on these DEGs has partially demonstrated that transcription factor forkhead box 3 and hepatocyte growth factor are highly expressed in tumors and are closely associated with chemosensitivity, indicating that they may serve as indicators of prognosis and chemotherapy efficacy $(34,35)$. Despite a number of studies having reported that genomic stability has an important function in the development of tumor resistance (36-38), the function of the majority of chemoresistance-associated genes has yet to be revealed. The analysis in the present suggests that during the formation of tumor chemotherapy tolerance, DEGs have an important role 
Table I. Key genes involved in multiple KEGG pathways in four types of cancer.

Tumor type

Estrogen receptor-negative

breast cancer

Ovarian cancer

Rectal cancer

Gastric cancer
Key genes

AKT2, AKT3, MAPK3, PIK3CD, CBLB, PIK3R2, BRAF, MAP2K2, DVL3, CTBP1, EP300, DVL2, CREBBP, MDM2, PML2, VHL, XIAP, ELOB, DIAS2 BIRC2,SOCS3, SOCS1, ARAF, PRKACA SOS1, CALM3, CALM2, PRKCZ, INSR INS, RAPGEF1, CALM1, CALM6, PGF FGF7, FGF18, VEGFB, RAC1, ADCY1 EGFR, CTNNB1, ADCY8, HGF, FGF10, LPAR5, ITGB1, FGFR2, RASGRP2, GNAS, RALGDS, FGFR1, RALB, VEGFA, FGF12, ADCY2, LPAR3, ITGA2B, LPAR2, RASSF5

CDC42, AKT2, SOS2, PIK3CB, COL6A3, LAMC1, COL1A1, COL6A1, FN1, LAMB1, ITGA3, THBS2, ITGB4, COL4A1, LAMA4, ITGB1, ITGB5, ITGA1, ITGB6, ITGA1, ITGB6, ITGB8, ITGA2B, COL9A2, JUN, TNXB, FGF14, FGF12, RASGRF1, GNG12, FGF23, FGF18, FGF7, YWHAZ, CREB3L1, MDM2, YWHAB, ACTN1, SYK, PGF, HGF, MET

HLA-DQA1, HLA-DPA1, HLA-DRB5, HLA-DRB4, HLA-DQA2, HLA-DPB1, HLA-DRA, HLA-DRB3, HLA-DRB1, CCND2, ATM, CDKN1A, CDK2, CALR, TAP2 PDIA3, CALR

Genes in bold participate in $\geq 3$ KEGG pathways; other genes participate in two KEGG pathways. KEGG, Kyoto Encyclopedia of Genes and Genomes.

Table II. The gene with the highest degree from each module of four cancer types.

Tumor type

Genes with the highest degree

Estrogen receptor-negative

breast cancer

Ovarian cancer

Rectal cancer

Gastric cancer

\author{
ZMYM2,DCAF15,ZNF160,ERBB2, PARD3,ACAN, VANT, BAG6,PIK3C2A, \\ CNTROB, GTF3C2, ARPC5L, PDE2A, FASTK \\ PAK7, COL1A1, SEMA3A, NEAT1, FGF14, MALAT1, MYH14, WSB1 \\ RPAP3, MTPAP, HLA-DRB1, EIF5, RPS16, FBXO7 \\ RPL3
}
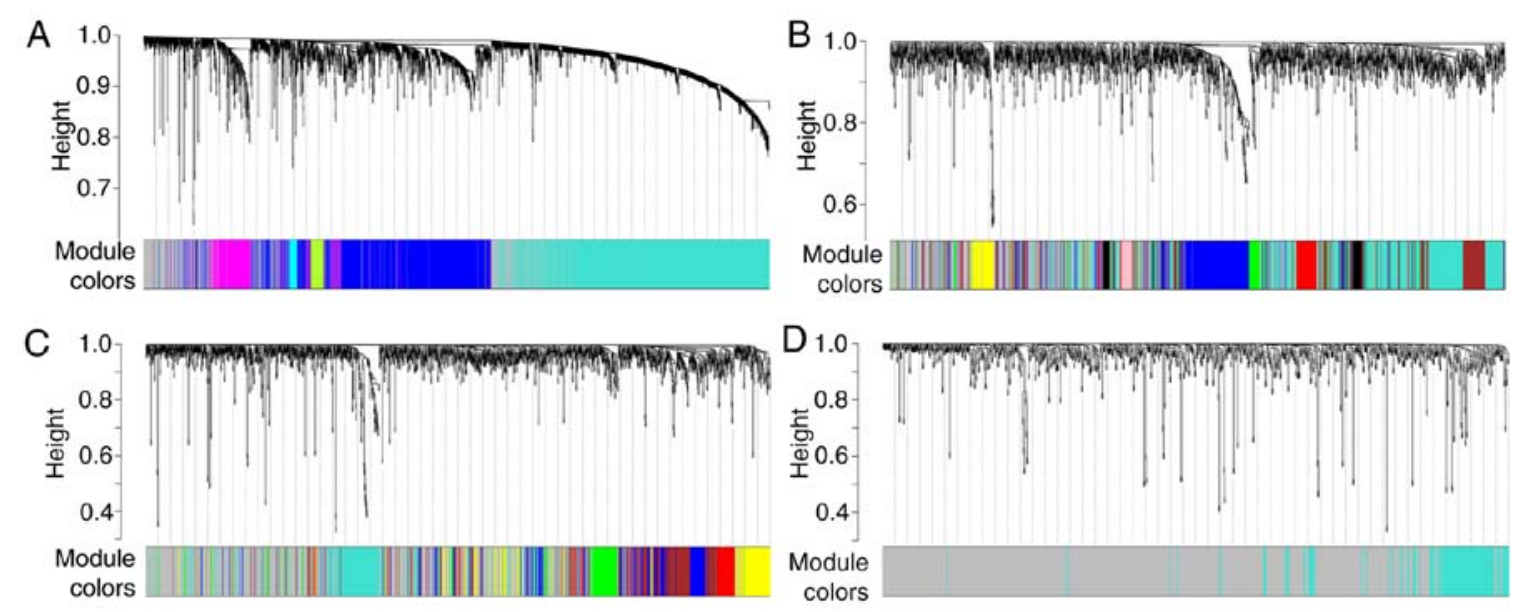

Figure 5. Clustering dendrogram of DEGs, with dissimilarity based on topological overlap, together with assigned module colors. The grey module represents a group of genes that have no study significance. (A) Estrogen receptor-negative breast cancer, (B) ovarian cancer, (C) rectal cancer and (D) gastric cancer.

in the most fundamental functions, including gene expression and regulation, providing further support of previous findings $(34,35)$. In addition, BP and KEGG pathway analyses of DEGs confirmed the important link between tumor immune function and chemoresistance. Previous studies have reported only individual mechanisms, for instance, macrophage-derived cathepsin regulation of tumor progression and direct regulation of tumor cell chemoresistance $(39,40)$. In addition, expression of $\mathrm{CXC}$ chemokine receptor 4 may contribute to tumor cell immune escape, potentially affecting tumor 
chemosensitivity (34). Based on the aforementioned results of previous studies, targeting the activity and pathways associated with DEGs via conventional chemotherapy is clinically important, since this approach may facilitate the combination with other immunotherapies to achieve maximum antitumor efficacy during clinical treatment.

Predictive networks have the potential to identify hundreds of genes that drive disease and can serve as points for therapeutic intervention (41). Our results offer a number of such gene targets. In KEGG analysis, it was indicated that multiple genes serve important roles in divergent signaling pathways, indicating that deregulation of these genes affects multiple tumor signatures, which may help in establishing the therapeutic potential of the target genes in these pathways in clinical settings. Furthermore, multiple PPI networks were constructed for different tumors using WGCNA and hub genes were screened via large-scale molecular analysis. The combined KEGG analysis and PPI network revealed that COL1A1, FGF14 and HLA-DRB1 are simultaneously involved in multiple signaling pathways and likely serve key roles in the development of chemoresistance. Furthermore, the current results suggest that a number of genes and signaling pathways have not yet been fully investigated and that their potential association with chemoresistance requires further validation. Further targeting of these genes in disease may, ultimately, involve targeting the entire network, as opposed to current therapeutic strategies that focus on one or two genes only.

In conclusion, the present study comprehensively examined the chemoresistance-associated genes and investigated the functions of these genes across multiple cancers based on RNA-Seq data. Despite that the biological significance of unreported DEGs in the development of tolerance to tumor chemotherapy warrants further evaluation, the findings of the present study propose a simple and effective strategy to identify genes involved in chemoresistance and to predict their potential functional roles, which may be useful for guiding subsequent experimental and therapeutic designs.

\section{Acknowledgements}

Not applicable.

\section{Funding}

This work was supported by the National Natural Science Foundation of China (grant no. 81572411).

\section{Availability of data and materials}

The datasets used during the present study are available from Gene Expression Omnibus (GSE16446, GSE30161, GSE45404 and GSE14209 at ncbi.nlm.nih.gov/geo/). The datasets used and/or analyzed during the current study are available from the corresponding author on reasonable request.

\section{Authors' contributions}

JG and WS conceived and designed the study. JY also participated in the design of the study and further conducted the data analysis. LT and ZY also participated in the data analysis. KT and GW checked the data, proposed suggestions for improvement and edited the manuscript. All authors read and approved the final manuscript.

\section{Ethics approval and consent to participate}

Not applicable.

\section{Patient consent for publication}

Not applicable.

\section{Competing interests}

The authors declare that they have no competing interests.

\section{References}

1. Zhang D and Fan D: New insights into the mechanisms of gastric cancer multidrug resistance and future perspectives. Future Oncol 6: 527-537, 2010.

2. Kathawala RJ, Gupta P, Ashby CR Jr and Chen ZS: The modulation of $\mathrm{ABC}$ transporter-mediated multidrug resistance in cancer: A review of the past decade. Drug Resist Updat 18: 1-17, 2015.

3. Holohan C, Van Schaeybroeck S, Longley DB and Johnston PG: Cancer drug resistance: An evolving paradigm. Nat Rev Cancer 13: 714-726, 2013.

4. Yook SH, Oltvai ZN and Barabási AL: Functional and topological characterization of protein interaction networks. Proteomics 4 : 928-942, 2004.

5. Barabási AL and Oltvai ZN: Network biology: Understanding the cell's functional organization. Nat Rev Genet 5: 101-113, 2004.

6. Desmedt C, Di Leo A, de Azambuja E, Larsimont D, Haibe-Kains B, Selleslags J, Delaloge S, Duhem C, Kains JP, Carly B, et al: Multifactorial approach to predicting resistance to anthracyclines. J Clin Oncol 29: 1578-1586, 2011.

7. Ferriss JS, Kim Y, Duska L, Birrer M, Levine DA, Moskaluk C, Theodorescu D and Lee JK: Multi-gene expression predictors of single drug responses to adjuvant chemotherapy in ovarian carcinoma: Predicting platinum resistance. PLoS One 7: e30550, 2012.

8. Agostini M, Zangrando A, Pastrello C, D'Angelo E, Romano G, Giovannoni R, Giordan M, Maretto I, Bedin C, Zanon C, et al: A functional biological network centered on XRCC3: A new possible marker of chemoradiotherapy resistance in rectal cancer patients. Cancer Biol Ther 16: 1160-1171, 2015.

9. Kim HK, Choi IJ, Kim CG, Kim HS, Oshima A, Michalowski A and Green JE: A gene expression signature of acquired chemoresistance to cisplatin and fluorouracil combination chemotherapy in gastric cancer patients. PLoS One 6: e16694, 2011.

10. Huang da W, Sherman BT and Lempicki RA: Bioinformatics enrichment tools: Paths toward the comprehensive functional analysis of large gene lists. Nucleic Acids Res 37: 1-13, 2009.

11. Huang da W, Sherman BT and Lempicki RA: Systematic and integrative analysis of large gene lists using DAVID bioinformatics resources. Nat Protoc 4: 44-57, 2009.

12. Langfelder P and Horvath S: WGCNA: An R package for weighted correlation network analysis. BMC Bioinformatics 9: 559,2008

13. Bindea G, Mlecnik B, Hackl H, Charoentong P, Tosolini M, Kirilovsky A, Fridman WH, Pagès F, Trajanoski $\mathrm{Z}$ and Galon J: ClueGO: A Cytoscape plug-in to decipher functionally grouped gene ontology and pathway annotation networks. Bioinformatics 25: 1091-1093, 2009.

14. Bindea G, Galon J and Mlecnik B: CluePedia Cytoscape plugin: Pathway insights using integrated experimental and in silico data. Bioinformatics 29: 661-663, 2013.

15. Du X, Zhao YP, Zhang TP, Zhou L, Chen G, Wang TX, You L and Shu H: Alteration of the intrinsic apoptosis pathway is involved in Notch-induced chemoresistance to gemcitabine in pancreatic cancer. Arch Med Res 45: 15-20, 2014.

16. Grossi V, Peserico A, Tezil T and Simone C: p38a MAPK pathway: A key factor in colorectal cancer therapy and chemoresistance. World J Gastroenterol 20: 9744-9758, 2014. 
17. Yuan J, Yin Z, Tao K, Wang G and Gao J: Function of insulin-like growth factor 1 receptor in cancer resistance to chemotherapy. Oncol Lett 15: 41-47, 2018.

18. Bystrykh L, Weersing E, Dontje B, Sutton S, Pletcher MT, Wiltshire T, Su AI, Vellenga E, Wang J, Manly KF, et al: Uncovering regulatory pathways that affect hematopoietic stem cell function using 'genetical genomics'. Nat Genet 37: 225-232, 2005.

19. Schadt EE, Lamb J, Yang X, Zhu J, Edwards S, Guhathakurta D, Sieberts SK, Monks S, Reitman M, Zhang C, et al: An integrative genomics approach to infer causal associations between gene expression and disease. Nat Genet 37: 710-717, 2005.

20. Yang Y, Han L, Yuan Y, Li J, Hei N and Liang H: Gene co-expression network analysis reveals common system-level properties of prognostic genes across cancer types. Nat Commun 5: 3231, 2014.

21. Marin JJ, Briz O, Monte MJ, Blazquez AG and Macias RI: Genetic variants in genes involved in mechanisms of chemoresistance to anticancer drugs. Curr Cancer Drug Targets 12: 402-438, 2012.

22. Delitto D, Vertes-George E, Hughes SJ, Behrns KE and Trevino JG: c-Met signaling in the development of tumorigenesis and chemoresistance: Potential applications in pancreatic cancer. World J Gastroenterol 20: 8458-8470, 2014.

23. Yuan X, Wu H, Xu H, Xiong H, Chu Q, Yu S, Wu GS and Wu K: Notch signaling: An emerging therapeutic target for cancer treatment. Cancer Lett 369: 20-27, 2015.

24. Sui X, Kong N, Ye L, Han W, Zhou J, Zhang Q, He C and Pan H: p38 and JNK MAPK pathways control the balance of apoptosis and autophagy in response to chemotherapeutic agents. Cancer Lett 344: 174-179, 2014

25. Racioppi L: CaMKK2: A novel target for shaping the androgen-regulated tumor ecosystem. Trends Mol Med 19: 83-88, 2013.

26. Lin F, Marcelo KL, Rajapakshe K, Coarfa C, Dean A, Wilganowski N, Robinson $\mathrm{H}$, Sevick E, Bissig KD Goldie LC, et al: The camKK2/camKIV relay is an essential regulator of hepatic cancer. Hepatology 62: 505-520, 2015.

27. Buemi M, Caccamo C, Nostro L, Cavallaro E, Floccari F and Grasso G: Brain and cancer: The protective role of erythropoietin. Med Res Rev 25: 245-259, 2005.

28. Wyman SK, Knouf EC, Parkin RK, Fritz BR, Lin DW, Dennis LM, Krouse MA, Webster PJ and Tewari M: Post-transcriptional generation of miRNA variants by multiple nucleotidyl transferases contributes to miRNA transcriptome complexity. Genome Res 21: 1450-1461, 2011.
29. Sepulveda JL and Wu C: The parvins. Cell Mol Life Sci 63 : 25-35, 2006.

30. Garrisi VM, Strippoli S, De Summa S, Pinto R, Perrone A, Guida G, Azzariti A, Guida M and Tommasi S: Proteomic profile and in silico analysis in metastatic melanoma with and without BRAF mutation. PLoS One 9: e112025, 2014.

31. Sun D, Liu Y, Zhang XS and Wu LY: CEA: Combination-based gene set functional enrichment analysis. Sci Rep 8: 13085, 2018

32. Lemmon MA and Schlessinger J: Cell signaling by receptor tyrosine kinases. Cell 141: 1117-1134, 2010.

33. Witsch E, Sela M and Yarden Y: Roles for growth factors in cancer progression. Physiology (Bethesda) 25: 85-101, 2010.

34. Sun J, Feng C, Liao W, Zhang H and Tang S: Expression of CXC chemokine receptor- 4 and forkhead box 3 in neuroblastoma cells and response to chemotherapy. Oncol Lett 7: 2083-2088, 2014.

35. Yang $\mathrm{H}$, Zhang $\mathrm{C}$ and Cui S: Expression of hepatocyte growth factor in breast cancer and its effect on prognosis and sensitivity to chemotherapy. Mol Med Rep 11: 1037-1042, 2015.

36. McGranahan N and Swanton C: Clonal heterogeneity and tumor evolution: Past, present, and the future. Cell 168: 613-628, 2017.

37. Dungrawala H, Bhat KP, Le Meur R, Chazin WJ, Ding X, Sharan SK, Wessel SR, Sathe AA, Zhao R and Cortez D: RADX promotes genome stability and modulates chemosensitivity by regulating RAD51 at replication forks. Mol Cell 67: 374-386.e5, 2017.

38. Juan HC, Lin Y, Chen HR and Fann MJ: Cdk12 is essential for embryonic development and the maintenance of genomic stability. Cell Death Differ 23: 1038-1048, 2016.

39. Mitchem JB, Brennan DJ, Knolhoff BL, Belt BA, Zhu Y, Sanford DE, Belaygorod L, Carpenter D, Collins L, Piwnica-Worms D, et al: Targeting tumor-infiltrating macrophages decreases tumor-initiating cells, relieves immunosuppression, and improves chemotherapeutic responses. Cancer Res 73: 1128-1141, 2013.

40. Genin M, Clement F, Fattaccioli A, Raes M and Michiels C: M1 and M2 macrophages derived from THP-1 cells differentially modulate the response of cancer cells to etoposide. BMC Cancer 15: 577, 2015.

41. Chen Y, Zhu J, Lum PY, Yang X, Pinto S, MacNeil DJ, Zhang C, Lamb J, Edwards S, Sieberts SK, et al: Variations in DNA elucidate molecular networks that cause disease. Nature 452: 429-435, 2008. 